CERIAS Tech Report 2005-03

\title{
SECURE OUTSOURCING OF SEQUENCE COMPARISONS
}

by Mikhail Atallah and Jiangtao Li

Center for Education and Research in

Information Assurance and Security,

Purdue University, West Lafayette, IN 47907-2086 


\title{
Secure Outsourcing of Sequence Comparisons *
}

\author{
Mikhail J. Atallah and Jiangtao Li \\ CERIAS and Department of Computer Sciences, Purdue University \\ 250 N. University Street, West Lafayette, IN 47907 \\ $\{m j a, j t l i\}$ ecs.purdue.edu
}

\begin{abstract}
Large-scale problems in the physical and life sciences are being revolutionized by Internet computing technologies, like grid computing, that make possible the massive cooperative sharing of computational power, bandwidth, storage, and data. A weak computational device, once connected to such a grid, is no longer limited by its slow speed, small amounts of local storage, and limited bandwidth: It can avail itself of the abundance of these resources that is available elsewhere on the network. An impediment to the use of "computational outsourcing" is that the data in question is often sensitive, e.g., of national security importance, or proprietary and containing commercial secrets, or to be kept private for legal requirements such as the HIPAA legislation, Gramm-Leach-Bliley, or similar laws. This motivates the design of techniques for computational outsourcing in a privacy-preserving manner, i.e., without revealing to the remote agents whose computational power is being used, either one's data or the outcome of the computation on the data. This paper investigates such secure outsourcing for widely applicable sequence comparison problems, and gives an efficient protocol for a customer to securely outsource sequence comparisons to two remote agents, such that the agents learn nothing about the customer's two private sequences or the result of the comparison. The local computations done by the customer are linear in the size of the sequences, and the computational cost and amount of communication done by the external agents are close to the time complexity of the best known algorithm for solving the problem on a single machine (i.e., quadratic, which is a huge computational burden for the kinds of massive data on which such comparisons are made). The sequence comparison problem considered arises in a large number of applications, including speech recognition, machine vision, and molecular sequence comparisons. In addition, essentially the same protocol can solve a larger class of problems whose standard dynamic programming solutions are similar in structure to the recurrence that subtends the sequence comparison algorithm.
\end{abstract}

\section{Introduction}

Internet computing technologies, like grid computing [8], enable a weak computational device connected to such a grid to be less limited by its inadequate local computational, storage, and bandwidth resources. However, such a weak computational device

\footnotetext{
* Portions of this work were supported by Grants IIS-0325345, IIS-0219560, IIS-0312357, and IIS-0242421 from the National Science Foundation, Contract N00014-02-1-0364 from the Office of Naval Research, by sponsors of the Center for Education and Research in Information Assurance and Security, and by Purdue Discovery Park's e-enterprise Center.
} 
(PDA, smartcard, sensor, etc) often cannot avail itself of the abundant resources available on the network because its data is sensitive. A prime example of this is DNA sequence comparisons: They are expensive enough to warrant remotely using the computing power available at powerful remote servers and super-computers, yet sensitive enough to give pause to anyone concerned that some unscrupulous person at the remote site may leak the DNA sequences or the comparison's outcome, or may subject the DNA to a battery of unauthorized tests whose outcome could have such grave consequences as jeopardizing an individual's insurability, employability, etc. Techniques for outsourcing expensive computational tasks in a privacy-preserving manner, are therefore an important research goal. This paper is a step in this direction, in that it gives a protocol for the secure outsourcing of the most important sequence comparison computation: The "string editing" problem, i.e., computing the edit-distance between two strings. The edit distance is one of the most widely used notions of similarity: It is the least-cost set of insertions, deletions, and substitutions required to transform one string into the other. Essentially the same protocol can solve the larger class of comparisons whose standard dynamic programming solution is similar in structure to that of string editing. The generalizations of edit distance that are solved by the same kind of dynamic programming recurrence relation as the one for edit distance, cover an even wider domain of applications. We use string editing here merely as the prototypical solution for this general class of dynamic programming recurrences.

In various ways and forms, sequence comparisons arise in many applications other than molecular sequence comparison, notably, in text editing, speech recognition, machine vision, etc. In fact the dynamic programming solution to this problem was independently discovered by no fewer than fourteen different researchers [22], and is given a different name by each discipline where it was independently discovered (NeedlemanWunsch by biologists, Wagner-Fischer by computer scientists, etc). For this reason, these problems have been studied rather extensively in the past, and form the object of several papers $[13,14,17,21,24,22,27]$, to list a few). The problems are typically solved by a serial algorithm in $\Theta(m n)$ time and space, through dynamic programming (cf. for example, [27]). When huge sequences are involved, the quadratic time complexity of the problem quickly becomes prohibitively expensive, requiring considerable power. Such super-computing power is widely available, but sending the data to such remote agents is problematic if the sequence data is sensitive, the outcome of the comparison is to be kept private, or both. In such cases, one can make a case for a technology that makes it possible for the customer to have the problem solved remotely but without revealing to the remote super-computing sites either the inputs to the computation or its outcome.

In other words we assume that Carol has two private sequences $\lambda$ and $\mu$, and wants to compute the similarity between these two sequences. Carol only has a weak computational device that is incapable of performing the sequence comparison locally. In order to get the result, Carol has to outsource the computation task to some external entities, the agents. If Carol trusted the agents, she could send the sequences directly to the external agents and ask them to compute the similarity on her behalf. However, if Carol is concerned about privacy, it is not acceptable to send the sequences to external agents because this would reveal too much information to these agents - both the sequences 
and the result. Our result is a protocol that computes the similarity of the sequences yet inherently safeguards the privacy of Carol's data. Assuming the two external agents do not conspire with each other against Carol by sharing the data that she sends to them, they learn nothing about the actual data and actual result.

The dynamic programming recurrence relation that subtends the solution to this problem, also serves to solve many other important related problems (either as special cases, or as generalizations that have the same dynamic programming kind of solution). These include the longest common subsequence problem, and the problem of approximate matching between a pattern sequence and text sequence (there is a huge literature of published work for the notion of approximate pattern matching and its connection to the sequence alignment problem). Any solution to the general sequence comparison problem could also be used to solve these related problems. For example, our protocol can enable a weak PDA to securely outsource the computation of the Unix command

$$
\text { diff file1 file2 | wc }
$$

to two agents where the agents learn nothing about file1, file2, and the result.

We now more precisely state the edit distance problem, in which the cost of an insertion or deletion or substitution is a symbol-dependent non-negative weight, and the edit distance is then the least-cost set of insertions, deletions, and substitutions required to transform one string into the other. More formally, if we let $\lambda$ be a string of length $n$, $\lambda=\lambda_{1} \ldots \lambda_{n}$ and $\mu$ be a string of length $m, \mu=\mu_{1} \ldots \mu_{m}$, both over some alphabet $\Sigma$. There are three types of allowed edit operations to be done on $\lambda$ : insertion of a symbol, deletion of a symbol, and substitution of one symbol by another. Each operation has a cost associated with it, namely $I(a)$ denotes the cost of inserting the symbol $a, D(a)$ denotes the cost of deleting $a$, and $S(a, b)$ denotes the cost of substituting $a$ with $b$. Each sequence of operations that transforms $\lambda$ into $\mu$ has a cost associated with it (which is equal to the sum of the costs of the operations in it), and the least-cost of such sequence is the edit-distance. The edit path is the actual sequence of operations that corresponds to the edit distance. Our outsourcing solution allows arbitrary $I(a), D(b)$, and $S(a, b)$ values, and we give better solutions for two special cases: (i) $S(a, b)=|a-b|$, and (ii) unit insertion/deletion cost and $S(a, b)=0$ if $a=b$ and $S(a, b)=+\infty$ if $a \neq b$ (in effect forbidding substitutions).

The rest of paper is organized as follows. We begin with a brief introduction of previous work in Section 2. Then we describe some building blocks in Section 3. In Section 4 , we present the secure outsourcing protocol for computing string edit distance. Section 5 extends the protocol so as to compute the edit path. Section 6 concludes.

\section{Related Work}

Recently, Atallah, Kerschbaum, and Du [2] developed an efficient protocol for sequence comparisons in the secure two-party computation framework in which each party has a private string; the protocol enables two parties to compute the edit distance of two sequences such that neither party learns anything about the private sequence of the other party. They [2] use dynamic programming to compare sequences, but in an additively split way - each party maintains a matrix, the summation of two matrices is the real 
matrix implicitly used to compute edit distance. Our protocol directly builds on their work, but is also quite different and more difficult in the following ways:

- We can no longer afford to have the customer carry out quadratic work or communication: Whereas in [2] there was "balance" in that all participants had equal computational and communication power, in our case the participant to whom all of the data and answer belong is asymmetrically weaker and is limited to a linear amount of computation and communication (hence cannot directly participate or help in each step of the quadratic-complexity dynamic programming solution).

- An even more crucial difference is the special difficulty this paper's framework faces in dealing with the costs table, that is, the table that contains the costs of deleting a symbol, inserting a symbol, and substituting one symbol by another: There is a quadratic number of accesses to this table, and the external agents cannot be allowed to learn which entry of the table is being consulted (because that would leak information about the inputs), yet the input owner's help cannot be enlisted for such table accesses because there is a quadratic number of them (recall that the owner is limited to linear work and communication - which is unavoidable).

Secure outsourcing of sequence comparisons adds to a growing list of problems considered in this framework (e.g. [4, 10,12, 15, 19,3], and others). We briefly review these next. In the server-aided secret computation literature (e.g. [4, 10,12,15, 19], to list a few), a weak smartcard performs public key encryptions by "borrowing" computing power from an untrusted server, without revealing to that server its private information. These papers deal primarily with the important problem of modular exponentiations. The paper [3] deals primarily with outsourcing of scientific computations.

In the the privacy homomorphism approach proposed in [20], the outsourcing agent is used as a permanent repository of data, performing certain operations on it and maintaining certain predicates, whereas the customer needs only to decrypt the data from the agent to obtain the real data; the secure outsourcing framework differs in that the customer is not interested in keeping data permanently with the external agents, instead, the customer only wants to temporarily use their superior computational power.

Du and Atallah have developed several models for secure remote database access with approximate matching [6]. One of the models that is related to our work is the secure storage outsourcing model where a customer who lacks storage space outsources her database to an external agent. The customer needs to query her database from time to time without revealing to the agent the queries and the results. Several protocols for other distance metrics were given, including Hamming distance, the $L_{1}$ and $L_{2}$ distance metrics. All these metrics considered in [6] were between strings that have the same length as each other - it is indeed a limitation of the techniques in [6] that they do not extend to the present situation where the strings are of different length and insertions and deletions are part of the definition. This makes the problem substantially different, as the edit distance algorithm is described by a dynamic program that computes it, rather than as a simple one-line mathematical expression to be securely computed. 


\section{Preliminaries}

Giving the full-fledged protocol would make it too long and rather hard to comprehend. This section aims at making the later presentation of the protocol much crisper by presenting some of the ideas and building blocks for it ahead of time, right after a brief review of the standard dynamic programming solution to string edit.

\subsection{Review of Edit Distance via Dynamic Programming}

We first briefly review the standard dynamic programming algorithm for computing edit distance. Let $M(i, j),(0 \leq i \leq n, 0 \leq j \leq m)$ be the minimum cost of transforming the prefix of $\lambda$ of length $i$ into the prefix of $\mu$ of length $j$, i.e., of transforming $\lambda_{1} \ldots \lambda_{i}$ into $\mu_{1} \ldots \mu_{j}$. Then $M(0,0)=0, M(0, j)=\sum_{k=1}^{j} I\left(\mu_{j}\right)$ for $1 \leq j \leq m, M(i, 0)=$ $\sum_{k=1}^{i} D\left(\lambda_{i}\right)$ for $1 \leq i \leq n$, and for positive $i$ and $j$ we have

$M(i, j)=\min \left(M(i-1, j-1)+S\left(\lambda_{i}, \mu_{j}\right), M(i-1, j)+D\left(\lambda_{i}\right), M(i, j-1)+I\left(\mu_{j}\right)\right)$

for all $i, j, 1 \leq i \leq n$ and $1 \leq j \leq m$. Hence $M(i, j)$ can be evaluated row-by-row or column-by-column in $\Theta(m n)$ time [27]. Observe that, of all entries of the $M$-matrix, only the three entries $M(i-1, j-1), M(i-1, j)$ and $M(i, j-1)$ are involved in the computation of the final value of $M(i, j)$.

Not only does the above dynamic program for computing $M$ depend on both $\lambda$ and $\mu$, but even if $M$ could be computed without knowing $\lambda$ and $\mu$, the problem remains that $M$ itself is too revealing: It reveals not only the overall edit distance, but also the edit distance from every prefix of $\lambda$ to every prefix of $\mu$. It is required in our problem that the external agents should learn nothing about the actual sequences and the results. The matrix $M$ should therefore not be known to the agents. It can of course not be stored at the customer's site, as it is a requirement that the customer is limited to $O(m+n)$ time and storage space.

\subsection{Framework}

We use two non-colluding agents in our protocol. Both the input sequences $(\lambda$ and $\mu)$ and the intermediate results (the matrix $M$ ) are additively split between the two agents, in such a way that neither one of the agents learns anything about the real inputs and results, but the two agents together can implicitly use the matrix $M$ without knowing it, that is, obtaining additively split answers "as if" they knew $M$. They have to do so without the help of the customer, as the customer is incapable of quadratic computation time or storage space. More details, about how this is done, are given below.

In the rest of the paper, we use following notations: We use $\mathcal{C}$ to denote the customer, $\mathcal{A}_{1}$ the first agent, and $\mathcal{A}_{2}$ the second agent. Any items superscripted with ' are known to $\mathcal{A}_{1}$ but not to $\mathcal{A}_{2}$, those superscripted with " are known to $\mathcal{A}_{2}$ but not to $\mathcal{A}_{1}$. In what follows, we often additively split an item $x$ between the two agents $\mathcal{A}_{1}$ and $\mathcal{A}_{2}$, i.e., we assume that $\mathcal{A}_{1}$ has an $x^{\prime}$ and $\mathcal{A}_{2}$ has an $x^{\prime \prime}$ such that $x=x^{\prime}+x^{\prime \prime}$; we do this splitting for the purpose of hiding $x$ from either agent. If arithmetic is modular, then this kind of additive splitting of $x$ hides it, in an information-theoretic sense, from $\mathcal{A}_{1}$ and $\mathcal{A}_{2}$. 
If, however, arithmetic is not modular, then even when $x^{\prime}$ and $x^{\prime \prime}$ can be negative and are very large compared to $x$, the "hiding" of $x$ is valid in a practical but not in an information-theoretic sense.

Splitting $\lambda$ and $\mu$ Let $\lambda$ and $\mu$ be two sequences over some finite alphabet $\Sigma=$ $\{0, \ldots, \sigma-1\}$. This could be a known fixed set of symbols (e.g., in biology $\Sigma=$ $\{A, C, T, G\}$ ), or the domain of a hash function that maps a potentially infinite alphabet into a finite domain. $\mathcal{C}$ splits $\lambda$ into $\lambda^{\prime}$ and $\lambda^{\prime \prime}$ such that $\lambda^{\prime}$ and $\lambda^{\prime \prime}$ are over the same alphabet $\Sigma$, and their sum is $\lambda$, i.e., $\lambda_{i}=\lambda_{i}^{\prime}+\lambda_{i}^{\prime \prime} \bmod \sigma$ for all $1 \leq i \leq n$. To split $\lambda, \mathcal{C}$ can first generate a random sequence $\lambda^{\prime}$ of length $n$, then set $\lambda_{i}^{\prime \prime}=\lambda_{i}-\lambda_{i}^{\prime} \bmod \sigma$ for all $1 \leq i \leq n$. Similarly, $\mathcal{C}$ splits $\mu$ into $\mu^{\prime}$ and $\mu^{\prime \prime}$ such that $\mu_{i}=\mu_{i}^{\prime}+\mu_{i}^{\prime \prime} \bmod \sigma$ for all $1 \leq i \leq m$. In the edit distance protocol, $\mathcal{C}$ sends $\lambda^{\prime}$ and $\mu^{\prime}$ to $\mathcal{A}_{1}$ and sends $\lambda^{\prime \prime}$ and $\mu^{\prime \prime}$ to $\mathcal{A}_{2}$.

Splitting $M$ Our edit distance protocol computes the same matrix as the dynamic programming algorithm, in the same order (e.g., row by row). Similar to [2], the matrix $M$ in our protocol is additively shared between $\mathcal{A}_{1}$ and $\mathcal{A}_{2}: \mathcal{A}_{1}$ and $\mathcal{A}_{2}$ each hold a matrix $M^{\prime}$ and $M^{\prime \prime}$, respectively, the sum of which is the matrix $M$, i.e., $M=M^{\prime}+$ $M^{\prime \prime}$; the protocol will maintain this property as an invariant through all its steps. The main challenge in our protocol is that the comparands and outcome of each comparison, as well as the indices of the minimum elements, have to be shared (in the sense that neither party individually knows them).

Hiding the sequences' lengths Splitting a sequence effectively hides its content, but fails to hide its length. In some situations, even the lengths of the sequences are sensitive and must be hidden or, at least, somewhat obfuscated. We now briefly sketch how to pad the sequences and obtain new, longer sequences whose edit distance is the same as that between the original sentences. Let $\hat{m}$ and $\hat{n}$ be the respective new lengths (with padding); assume that randomly choosing $\hat{m}$ from the interval $[m, 2 m]$ provides enough obfuscation of $m$, and similarly $\hat{n}$ from the interval $[n, 2 n]$.

We introduce a new special symbol "\$" to the alphabet $\Sigma$ such that the cost of insertion and deletion of this symbol is 0 (i.e., $I(\$)=D(\$)=0$ ), and the cost of substitution of this symbol is infinity (i.e., $S(\$, a)=S(a, \$)=+\infty$ for every symbol $a$ in $\Sigma$ ). The customer appends " $\$$ "s to the end of $\lambda$ and $\mu$ to turn their respective lengths into the target values $\hat{n}$ and $\hat{m}$, before splitting and sending them to the agents. This padding has following two properties: 1) the edit distance between the padded sequences is the same as the edit distance between the original sequences, 2) the agents cannot figure out how many " $\$$ "s were padded into a sequence because of the random split of the sequence.

To avoid unnecessarily cluttering the exposition, we assume $\lambda$ and $\mu$ are already padded with " $\$$ "s before the protocol, thus we assume the lengths of $\lambda$ and $\mu$ are still $n$ and $m$ respectively, and the alphabet $\Sigma$ is still $\{0, \ldots, \sigma-1\}$. 


\subsection{Secure Table Lookup Protocol for Split Data}

Recall that the $\sigma \times \sigma$ size cost table $S$ is public, hence known to both $\mathcal{A}_{1}$ and $\mathcal{A}_{2}$; we make no assumptions about the costs in the table (they can be arbitrary, not necessarily between 0 and $\sigma-1$ ). Recall that $\mathcal{A}_{1}$ and $\mathcal{A}_{2}$ share additively each symbol $\alpha$ from $\lambda$ and $\beta$ from $\mu$, i.e., $\alpha=\alpha^{\prime}+\alpha^{\prime \prime} \bmod \sigma$, and $\beta=\beta^{\prime}+\beta^{\prime \prime} \bmod \sigma$ where $\mathcal{A}_{1}$ has $\alpha^{\prime}$ and $\beta^{\prime}, \mathcal{A}_{2}$ has $\alpha^{\prime \prime}$ and $\beta^{\prime \prime} . \mathcal{A}_{1}$ and $\mathcal{A}_{2}$ want to cooperatively look up the value $S(\alpha, \beta)$ from the cost table $S$, but without either of them knowing which entry of $S$ was accessed and what value was returned by the access (so that value itself must be additively split). The protocol below solves this lookup problem in one round and $O\left(\sigma^{2}\right)$ computation and communication; note that naively using the protocol below $O(m n)$ times would result in an $O\left(\sigma^{2} m n\right)$ computation and communication complexity for the overall sequence comparison problem, not the $O(\sigma m n)$ performance we claim (and that will be substantiated later in the paper).

Protocol 1 Secure Table Lookup Protocol

Input $\mathcal{A}_{1}$ has $\alpha^{\prime}$ and $\beta^{\prime}$ and $\mathcal{A}_{2}$ has $\alpha^{\prime \prime}$ and $\beta^{\prime \prime}$, such that $\alpha=\alpha^{\prime}+\alpha^{\prime \prime} \bmod \sigma$ and $\beta=\beta^{\prime}+\beta^{\prime \prime} \bmod \sigma$.

Output $\mathcal{A}_{1}$ obtains a number $a$, and $\mathcal{A}_{2}$ obtains a number $b$, such that $a+b=S(\alpha, \beta)$.

The protocol steps are:

1. $\mathcal{A}_{1}$ generates a key pair for a homomorphic semantically-secure public key system and sends the public key to $\mathcal{A}_{2}$ (any of the existing systems will do, e.g., $[16,18]$ ). In what follows $E(\cdot)$ denotes encryption with $\mathcal{A}_{1}$ 's public key, and $D(\cdot)$ decryption with $\mathcal{A}_{1}$ 's private key. (Recall that the homomorphic property implies that $E(x) *$ $E(y)=E(x+y)$, and semantic security implies that $E(x)$ reveals nothing about $x$, so that $x=y$ need not imply $E(x)=E(y)$.)

2. $\mathcal{A}_{1}$ generates a $\sigma \times \sigma$ size table $\hat{S}$ with entry $\hat{S}(i, j)$ equal to $E\left(S\left(i+\alpha^{\prime} \bmod \sigma, j+\right.\right.$ $\left.\left.\beta^{\prime} \bmod \sigma\right)\right)$ for all $0 \leq i, j \leq \sigma-1$, and sends that table $\hat{S}$ to $\mathcal{A}_{2}$.

3. $\mathcal{A}_{2}$ picks up the $\left(\alpha^{\prime \prime}, \beta^{\prime \prime}\right)$ th entry from the table received in the previous step, which is $\hat{S}\left(\alpha^{\prime \prime}, \beta^{\prime \prime}\right)=E(S(\alpha, \beta)) . \mathcal{A}_{2}$ then generates a random number $b$, then computes $\theta=E(S(\alpha, \beta)) * E(-b)=E(S(\alpha, \beta)-b)$, and sends it back to $\mathcal{A}_{1}$.

4. $\mathcal{A}_{1}$ decrypts the value received from $\mathcal{A}_{2}$ and gets $a=D(E(S(\alpha, \beta)-b))=$ $S(\alpha, \beta)-b$.

As required, $a+b=S(\alpha, \beta)$, and $\mathcal{A}_{1}$ and $\mathcal{A}_{2}$ do not learn anything about the other party from the protocol. The computation and communication cost of this protocol is $O\left(\sigma^{2}\right)$.

\section{Edit Distance Protocol}

We now "put the pieces together" and give the overall protocol. We begin with the general case of arbitrary $I(a), D(b), S(a, b)$. Then two special cases are considered. One is the case of arbitrary $I(a)$ and $D(b)$, but $S(a, b)=|a-b|$. The other is the practical case of unit insertion/deletion cost and forbidden substitutions (i.e., $S(a, b)$ is 0 if $a=b$ and 
$+\infty$ otherwise). For all the above cases, the cost of computation and communication by the customer is linear to the size of the input. The cost of computation and communication by agents is $O(\sigma m n)$ for the general case and $O(m n)$ for the two special cases.

\subsection{The General Case: Arbitrary $I(a), D(b), S(a, b)$}

In this section, we begin with a preliminary solution that is not our best, but serves as a useful "warmup" to the more efficient solution that comes later in this section.

A preliminary version of the protocol Recall that $\mathcal{C}$ splits $\lambda$ into $\lambda^{\prime}$ and $\lambda^{\prime \prime}$ and $\mu$ into $\mu^{\prime}$ and $\mu^{\prime \prime}$, then sends $\lambda^{\prime}$ and $\mu^{\prime}$ to $\mathcal{A}_{1}$, and sends $\lambda^{\prime \prime}$ and $\mu^{\prime \prime}$ to $\mathcal{A}_{2} . \mathcal{A}_{1}$ and $\mathcal{A}_{2}$ each maintains a matrix $M^{\prime}$ and (respectively) $M^{\prime \prime}$, such that $M=M^{\prime}+M^{\prime \prime} . \mathcal{A}_{1}$ and $\mathcal{A}_{2}$ compute each element $M(i, j)$ in additively split fashion; this is done as prescribed in the recursive edit distance formula, by $\mathcal{A}_{1}$ and $\mathcal{A}_{2}$ updating their respective $M^{\prime}$ and $M^{\prime \prime}$. After doing so, $\mathcal{A}_{1}$ and $\mathcal{A}_{2}$, send their respective $M^{\prime}(n, m)$ and $M^{\prime \prime}(n, m)$ back to $\mathcal{C}$. $\mathcal{C}$ can then obtain the edit distance $M(n, m)=M^{\prime}(n, m)+M^{\prime \prime}(n, m)$.

During the computation of each element $M(i, j), S\left(\lambda_{i}, \mu_{j}\right)$ has to be computed by $\mathcal{A}_{1}$ and $\mathcal{A}_{2}$ in additively split fashion and without the help of $\mathcal{C}$, which implies that the substitution table $S$ should be known by both $\mathcal{A}_{1}$ and $\mathcal{A}_{2}$. Hence, $\mathcal{C}$ needs to send the table to both of the agents during the initialization phase of the protocol. The content of the table is not private, and need not be disguised.

Initialization of Matrices $M^{\prime}$ and $M^{\prime \prime}$ should be initialized so that their sum $M$ has $M(0, j)$ and $M(i, 0)$ equal to the values specified in Section 3.1. The $M(i, j)$ entries for nonzero $i$ and $j$ can be random (they will be computed later, after the initialization). The following initializes the $M^{\prime}$ and $M^{\prime \prime}$ matrices:

1. $\mathcal{C}$ generates two vectors of random numbers $\boldsymbol{a}=\left(a_{1}, \ldots, a_{n}\right)$ and $\boldsymbol{b}=\left(b_{1}, \ldots, b_{m}\right)$. Then $\mathcal{C}$ computes two vectors $\boldsymbol{c}=\left(c_{1}, \ldots, c_{n}\right)$ and $\boldsymbol{d}=\left(d_{1}, \ldots, d_{m}\right)$ where

(a) $c_{i}=\sum_{k=1}^{i} D\left(\lambda_{k}\right)-a_{i}$ for $1 \leq i \leq n$,

(b) $d_{j}=\sum_{k=1}^{j} I\left(\mu_{k}\right)-b_{j}$ for $1 \leq j \leq m$.

$\mathcal{C}$ sends to $\mathcal{A}_{1}$ the vectors $\boldsymbol{b}, \boldsymbol{c}$, and to $\mathcal{A}_{2}$ the vectors $\boldsymbol{a}, \boldsymbol{d}$.

2. $\mathcal{A}_{1}$ sets $M^{\prime}(0, j)=b_{j}$ for $1 \leq j \leq m$, and sets $M^{\prime}(i, 0)=c_{i}$ for $1 \leq i \leq n$. All the other entries of $M^{\prime}$ are set to 0 .

3. $\mathcal{A}_{2}$ sets $M^{\prime \prime}(i, 0)=a_{i}$ for $1 \leq i \leq n$, and sets $M^{\prime \prime}(0, j)=d_{j}$ for $1 \leq j \leq m$. All the other entries of $M^{\prime \prime}$ are set to 0 .

Note that the above does implicitly initialize $M(i, j)$ in the correct way, because it results in

$$
\begin{aligned}
& \text { - } M^{\prime}(0,0)+M^{\prime \prime}(0,0)=0 . \\
& \text { - } M^{\prime}(0, j)+M^{\prime \prime}(0, j)=\sum_{k=1}^{j} I\left(\mu_{j}\right) \text { for } 1 \leq j \leq m . \\
& \text { - } M^{\prime}(i, 0)+M^{\prime \prime}(i, 0)=\sum_{k=1}^{i} D\left(\lambda_{i}\right) \text { for } 1 \leq i \leq n .
\end{aligned}
$$

Neither $\mathcal{A}_{1}$ nor $\mathcal{A}_{2}$ gain any information about $\lambda$ and $\mu$ from the initialization of their matrices, because the two vectors they each receive from $\mathcal{C}$ look random to them. 
Mimicking a step of the dynamic program The following protocol describes how an $M(i, j)$ computation is done by $\mathcal{A}_{1}$ and $\mathcal{A}_{2}$, i.e., how they modify their respective $M^{\prime}(i, j)$ and $M^{\prime \prime}(i, j)$, thus implicitly computing the final $M(i, j)$ without either of them learning which update was performed.

1. $\mathcal{A}_{1}$ and $\mathcal{A}_{2}$ use the secure table lookup protocol with inputs $\lambda_{i}^{\prime}$ and $\mu_{j}^{\prime}$ from $\mathcal{A}_{1}$, and inputs $\lambda_{i}^{\prime \prime}$ and $\mu_{j}^{\prime \prime}$ from $\mathcal{A}_{2}$. As a result, $\mathcal{A}_{1}$ obtains $\gamma^{\prime}$ and $\mathcal{A}_{2}$ obtains $\gamma^{\prime \prime}$ such that

$$
\gamma^{\prime}+\gamma^{\prime \prime}=S\left(\lambda_{i}^{\prime}+\lambda_{i}^{\prime \prime} \bmod \sigma, \mu_{j}^{\prime}+\mu_{j}^{\prime \prime} \bmod \sigma\right)=S\left(\lambda_{i}, \mu_{j}\right) .
$$

$\mathcal{A}_{1}$ then forms $u^{\prime}=M^{\prime}(i-1, j-1)+\gamma^{\prime}$ and Bob forms $u^{\prime \prime}=M^{\prime \prime}(i-1, j-1)+\gamma^{\prime \prime}$. Observe that $u^{\prime}+u^{\prime \prime}=M(i-1, j-1)+S\left(\lambda_{i}, \mu_{j}\right)$, which is one of the three quantities involved in the update step for $M(i, j)$ in the dynamic program.

2. $\mathcal{A}_{1}$ computes $v^{\prime}=M^{\prime}(i-1, j)+M^{\prime}(i, 0)-M^{\prime}(i-1,0)=M^{\prime}(i-1,0)+$ $D\left(\lambda_{i}\right)-a_{i}+a_{i-1}, \mathcal{A}_{2}$ computes $v^{\prime \prime}=M^{\prime \prime}(i-1, j)+M^{\prime \prime}(i, 0)-M^{\prime \prime}(i-1,0)=$ $M^{\prime \prime}(i-1, j)+a_{i}-a_{i-1}$. Observe that $u_{A}+u_{B}=M(i-1, j)+D\left(\lambda_{i}\right)$, which is one of the three quantities involved in the update step for $M(i, j)$ in the dynamic program.

3. $\mathcal{A}_{1}$ computes $w^{\prime}=M^{\prime}(i, j-1)+M^{\prime}(0, j)-M^{\prime}(0, j-1)=M^{\prime}(i, j-1)+b_{j}-b_{j-1}$, $\mathcal{A}_{2}$ computes $w^{\prime \prime}=M^{\prime \prime}(i, j-1)+M^{\prime \prime}(0, j)-M^{\prime \prime}(0, j-1)=M^{\prime \prime}(i, j-1)+$ $I\left(\mu_{j}\right)-b_{j}+b_{j-1}$. Observe that $w^{\prime}+w^{\prime \prime}=M(i, j-1)+D\left(\mu_{j}\right)$, which is one of the three quantities involved in the update step for $M(i, j)$ in the dynamic program.

4. $\mathcal{A}_{1}$ and $\mathcal{A}_{2}$ use the minimum finding protocol for split data (described in [2]) on their respective vectors $\left(u^{\prime}, v^{\prime}, w^{\prime}\right)$ and $\left(u^{\prime \prime}, v^{\prime \prime}, w^{\prime \prime}\right)$. As a result, $\mathcal{A}_{1}$ gets an $x^{\prime}$ and $\mathcal{A}_{2}$ gets an $x^{\prime \prime}$ whose sum $x^{\prime}+x^{\prime \prime}$ is

$$
\begin{gathered}
\min \left(u^{\prime}+u^{\prime \prime}, v^{\prime}+v^{\prime \prime}, w^{\prime}+w^{\prime \prime}\right)= \\
\min \left(M(i-1, j-1)+S\left(\lambda_{i}, \mu_{j}\right), M(i-1, j)+D\left(\lambda_{i}\right), M(i, j-1)+I\left(\mu_{j}\right)\right) .
\end{gathered}
$$

5. $\mathcal{A}_{1}$ sets $M^{\prime}(i, j)$ equal to $x^{\prime}$, and $\mathcal{A}_{2}$ sets $M^{\prime \prime}(i, j)$ equal to $x^{\prime \prime}$.

Performance Analysis The local computations done by $\mathcal{C}$ in the above protocol consist of splitting $\lambda$ and $\mu$ and sending the resulting shares to the agents, and computing and sending the vectors $\boldsymbol{a}, \boldsymbol{b}, \boldsymbol{c}, \boldsymbol{d}$. These are done in $O(m+n)$ time and communication.

Each agent mimics $m n$ steps of the dynamic program. During each step, two agents run the secure table lookup protocol once and the minimum finding protocol once. Thus, the communication between $\mathcal{A}_{1}$ and $\mathcal{A}_{2}$ for each such step is $O\left(\sigma^{2}\right)+O(1)$. Therefore the total computation and communication cost for each agent is $O\left(\sigma^{2} m n\right)$.

An improved version of the protocol A bottleneck in the above protocol is the split computation of $S\left(\lambda_{i}, \mu_{j}\right)$ : Running the secure table lookup protocol at each step of the dynamic program costs an expensive $O\left(\sigma^{2}\right)$. In this subsection, we present a solution that is more efficient by a factor of $\sigma$.

Recall that in the dynamic program, $M$ is constructed row-by-row or column-bycolumn. We assume, without loss of generality that $M$ is computed row-by-row. We will compute $S\left(\lambda_{i}, \mu_{j}\right)$ row-by-row exploiting the fact that all $\left(\lambda_{i}, \mu_{j}\right)$ in row $i$ have the same $\lambda_{i}$ : We will "batch" these table accesses for row $i$, as we describe next. 


\section{Protocol 2 Batched Secure Table Lookup Protocol}

Input $\mathcal{A}_{1}$ has $\lambda_{i}^{\prime}$ and $\mu^{\prime}=\mu_{1}^{\prime}, \ldots, \mu_{m}^{\prime}$, and $\mathcal{A}_{2}$ has $\lambda_{i}^{\prime \prime}$ and $\mu^{\prime \prime}=\mu_{1}^{\prime \prime}, \ldots, \mu_{m}^{\prime \prime}$, all symbols being over alphabet $\Sigma$.

Output $\mathcal{A}_{1}$ and $\mathcal{A}_{2}$ each obtains a vector $\gamma^{\prime}$ and $\gamma^{\prime \prime}$ of size $m$, such that $\gamma_{j}^{\prime}+\gamma_{j}^{\prime \prime}=$ $S\left(\lambda_{i}, \mu_{j}\right)$ for $1 \leq j \leq m$.

The protocol is:

1. $\mathcal{A}_{1}$ generates a key pair for a homomorphic semantically-secure public key system and sends the public key to $\mathcal{A}_{2}$. As before, $E(\cdot)$ denotes encryption with $\mathcal{A}_{1}$ 's public key, and $D(\cdot)$ decryption with $\mathcal{A}_{1}$ 's private key.

2. $\mathcal{A}_{1}$ generates a $\sigma \times \sigma$ table $\hat{S}$ with $\hat{S}(k, l)$ equal to $E\left(S\left(k+\lambda_{i}^{\prime} \bmod \sigma, l\right)\right)$ for all $0 \leq k, l \leq \sigma-1$, and sends that table to $\mathcal{A}_{2}$.

3. For each $j=1, \ldots, m$, the next 5 sub-steps are carried out to compute the $\left(\gamma_{j}^{\prime}, \gamma_{j}^{\prime \prime}\right)$ pair.

(a) $\mathcal{A}_{2}$ creates a $\sigma$ size vector $\boldsymbol{v}$ equal to the $\lambda_{i}^{\prime \prime}$ th row of the table $\hat{S}$ received in the previous step. Observe that $v_{l}=E\left(S\left(\lambda_{i}^{\prime \prime}+\lambda_{i}^{\prime} \bmod \sigma, l\right)\right)=E\left(S\left(\lambda_{i}, l\right)\right)$ for $0 \leq l \leq \sigma-1$.

(b) $\mathcal{A}_{2}$ circularly left-shifts $\boldsymbol{v}$ by $\mu_{j}^{\prime \prime}$ positions, so that $v_{l}$ becomes $E\left(S\left(\lambda_{i}, \mu_{j}^{\prime \prime}+\right.\right.$ $l \bmod \sigma))$ for $0 \leq l \leq \sigma-1$.

(c) $\mathcal{A}_{2}$ generates a random number $\gamma_{j}^{\prime \prime}$, he then updates $\boldsymbol{v}$ by setting $v_{l}=v_{l} *$ $E\left(-\gamma_{j}^{\prime \prime}\right)=E\left(S\left(\lambda_{i}, \mu_{j}^{\prime \prime}+l \bmod \sigma\right)-\gamma_{j}^{\prime \prime}\right)$ for $0 \leq l \leq \sigma-1$. Note that the $\mu_{j}^{\prime}$ th entry of the resulting $\boldsymbol{v}$ is now $E\left(S\left(\lambda_{i}, \mu_{j}\right)-\gamma_{j}^{\prime \prime}\right)$.

(d) $\mathcal{A}_{1}$ uses a 1-out-of- $\sigma$ oblivious transfer protocol to obtain the $\mu_{j}^{\prime}$ th entry of $v$ from $\mathcal{A}_{2}$ without revealing to $\mathcal{A}_{2}$ which $v_{l}$ he received (see, e.g., [23] for many detailed oblivious transfer protocols).

(e) $\mathcal{A}_{1}$ decrypts the value he obtained from the oblivious transfer of the previous step, and gets $\gamma_{j}^{\prime}=S\left(\lambda_{i}, \mu_{j}\right)-\gamma_{j}^{\prime \prime}$. Observe that $\gamma_{j}^{\prime}+\gamma_{j}^{\prime \prime}=S\left(\lambda_{i}, \mu_{j}\right)$, as required.

Neither $\mathcal{A}_{1}$ nor $\mathcal{A}_{2}$ learned anything about which entry of $S$ was implicitly accessed, or what the value obtained in split fashion is. The communication cost of the above scheme is $O\left(\sigma^{2}\right)+O(\sigma m)$. The size of the alphabet is much smaller than the length of a sequence (e.g., in bioinformatics $\sigma=4$ whereas a sequence's length is huge). Therefore the dominant term in the complexity of the above is $O(\sigma m)$.

The new outsourcing protocol for sequence comparisons is same as the preliminary protocol in the previous subsection, except for some modifications in the first step of the protocol, titled "mimicking a step of the dynamic program". Recall that the aim of Step 1 is to produce a $u^{\prime}$ with $\mathcal{A}_{1}$ and a $u^{\prime \prime}$ with $\mathcal{A}_{2}$ such that $u^{\prime}+u^{\prime \prime}=M(i-1, j-1)+$ $S\left(\lambda_{i}, \mu_{j}\right)$. In the improved protocol, we first run the above batched lookup protocol for row $i$ to produce a $\gamma^{\prime}$ for $\mathcal{A}_{1}$ and a $\gamma^{\prime \prime}$ for $\mathcal{A}_{2}$, such that $\gamma_{j}^{\prime}+\gamma_{j}^{\prime \prime}=S\left(\lambda_{i}, \mu_{j}\right)$ for $1 \leq j \leq$ $m$. Then, during Step 1 of the modified protocol, $\mathcal{A}_{1}$ sets $u^{\prime}=M^{\prime}(i-1, j-1)+\gamma_{j}^{\prime}$ and $\mathcal{A}_{2}$ sets $u^{\prime \prime}=M^{\prime \prime}(i-1, j-1)+\gamma_{j}^{\prime \prime}$. Note that, at the end of the new Step 1, $u^{\prime}+u^{\prime \prime}$ equals to $M(i-1, j-1)+S\left(\lambda_{i}, \mu_{j}\right)$, as required. The computational task for the customer in this protocol is the same as in the preliminary version. The computational and communication cost for the agents in this protocol are $\Theta(\sigma m n)$. 


\subsection{The Case $S(a, b)=|a-b|$}

The improvement in this case comes from a more efficient way of computing the split $S\left(\lambda_{i}, \mu_{j}\right)$ values needed in Step 1 of the protocol. Unlike previous sections of the paper, each symbol in $\lambda$ and $\mu$ is split into two numbers that are not modulo $\sigma$, and can in fact be arbitrary (and possibly negative) integers. The protocol is otherwise the same as in section 4.1.

The main difference is in the first step of sub-protocol "mimicking a step of the dynamic program". Note that

$$
\begin{aligned}
S\left(\lambda_{i}, \mu_{j}\right) & =\left|\lambda_{i}-\mu_{j}\right| \\
& =\max \left(\lambda_{i}-\mu_{j}, \mu_{j}-\lambda_{i}\right) \\
& =\max \left(\left(\lambda_{i}^{\prime}-\mu_{j}^{\prime}\right)+\left(\lambda_{i}^{\prime \prime}-\mu_{j}^{\prime \prime}\right),\left(\mu_{j}^{\prime}-\lambda_{i}^{\prime}\right)+\left(\mu_{j}^{\prime \prime}-\lambda_{i}^{\prime \prime}\right)\right)
\end{aligned}
$$

The $S\left(\lambda_{i}, \mu_{j}\right)$ can be computed as follows: $\mathcal{A}_{1}$ forms a two-entry vector $\boldsymbol{v}^{\prime}=\left(\lambda_{i}^{\prime}-\right.$ $\left.\mu_{j}^{\prime}, \mu_{j}^{\prime}-\lambda_{i}^{\prime}\right), \mathcal{A}_{2}$ forms a two-entry vector $\boldsymbol{v}^{\prime \prime}=\left(\lambda_{i}^{\prime \prime}-\mu_{j}^{\prime \prime}, \mu_{j}^{\prime \prime}-\lambda_{i}^{\prime \prime}\right)$, then $\mathcal{A}_{1}$ and $\mathcal{A}_{2}$ use the split maximum finding protocol (described in [2]) to obtain $\gamma^{\prime}$ and $\gamma^{\prime \prime}$ such that

$$
\gamma^{\prime}+\gamma^{\prime \prime}=\max \left(\boldsymbol{v}^{\prime}+\boldsymbol{v}^{\prime \prime}\right)=\left|\lambda_{i}-\mu_{j}\right|=S\left(\lambda_{i}, \mu_{j}\right) .
$$

Then the first step of the dynamic program can be replaced by $\mathcal{A}_{1}$ setting $u^{\prime}=M^{\prime}(i-$ $1, j-1)+\gamma^{\prime}$, and $\mathcal{A}_{2}$ setting $u^{\prime \prime}=M^{\prime \prime}(i-1, j-1)+\gamma^{\prime \prime}$. As required, $u^{\prime}+u^{\prime \prime}$ equals $M(i-1, j-1)+S\left(\lambda_{i}, \mu_{j}\right)$. Since the communication cost of Step 1 is now $O(1)$, the total communication cost for the agents is $O(m n)$.

\subsection{The Case of Unit Insertion/Deletion Costs and Forbidden Substitutions}

The improvement in this case directly follows from a technique, given in [2], that we now review. Forbidden substitutions means that $S(a, b)$ is $+\infty$ unless $a=b$ (in which case it is zero because it is a "do nothing" operation). Of course a substitution is useless if its cost is 2 or more (because one might as well achieve the same effect with a deletion followed by an insertion). The protocol is then:

1. For $i=\sigma, \ldots, 1$ in turn, $\mathcal{C}$ replaces every occurrence of symbol $i$ by the symbol $2 i$. So the alphabet becomes effectively $\{0,2,4, \ldots, 2 \sigma-2\}$.

2. $\mathcal{C}$ runs the protocol given in the previous section for the case of $S(a, b)=|a-b|$, using a unit cost for every insertion and every deletion.

The reason it works is that, after the change of alphabet, $S(a, b)$ is zero if $a=b$ and 2 or more if $a \neq b$, i.e., it is as if $S(a, b)=+\infty$ if $a \neq b$ (recall that a substitution is useless if its cost is 2 or more, because one can achieve the same effect with a deletion followed by an insertion).

\section{Computing the Edit Path}

We have so far established that the edit distance can be computed in linear space and $O(\sigma m n)$ time and communication. This section deals with extending this to computing, 
also in split form, the edit path, which is a sequence of operations that corresponds to the edit distance (that is, a minimum-cost sequence of operations on $\lambda$ that turns it into $\mu)$. We show that the edit path can be computed by the agents in split form in $O(m n)$ space and in $O(\sigma m n)$ time and communication.

\subsection{Review: Grid Graph View of the Problem}

The interdependencies among the entries of the $M$-matrix induce an $(n+1) \times(m+$ 1) grid directed acyclic graph (grid DAG for short) associated with the string editing problem. It is easy to see that in fact the string editing problem can be viewed as a shortest-paths problem on a grid DAG.

Definition 1. An $l_{1} \times l_{2}$ grid DAG is a directed acyclic graph whose vertices are the $l_{1} l_{2}$ points of an $l_{1} \times l_{2}$ grid, and such that the only edges from grid point $(i, j)$ are to grid points $(i, j+1),(i+1, j)$, and $(i+1, j+1)$.

Note that the top-left point of a grid DAG has no edge entering it (i.e., is a source), and that the bottom-right point has no edge leaving it (i.e., is a sink). We now review the correspondence between edit scripts and grid graphs. We associate an $(n+1) \times(m+1)$ grid DAG $G$ with the string editing problem in the natural way: The $(n+1)(m+1)$ vertices of $G$ are in one-to-one correspondence with the $(n+1)(m+1)$ entries of the $M$-matrix, and the cost of an edge from vertex $(k, l)$ to vertex $(i, j)$ is equal to $I\left(\mu_{j}\right)$ if $k=i$ and $l=j-1$, to $D\left(\lambda_{i}\right)$ if $k=i-1$ and $l=j$, to $S\left(\lambda_{i}, \mu_{j}\right)$ if $k=i-1$ and $l=j-1$. We can restrict our attention to edit paths which are not wasteful in the sense that they do no obviously inefficient moves such as: inserting then deleting the same symbol, or changing a symbol into a new symbol which they then delete, etc. More formally, the only edit scripts considered are those that apply at most one edit operation to a given symbol occurrence. Such edit scripts that transform $\lambda$ into $\mu$ or vice versa are in one-to-one correspondence to the weighted paths of $G$ that originate at the source (which corresponds to $M(0,0)$ ) and end on the sink (which corresponds to $M(n, m)$ ). Thus, any complexity bounds we establish for the problem of finding a shortest (i.e., least-cost) source-to-sink path in an $(n+1) \times(m+1)$ grid DAG $G$, extends naturally to the string editing problem.

At first sight it looks like "remembering" (in split form), for every entry $M(i, j)$, which of $\{M(i-1, j-1), M(i-1, j), M(i, j-1)\}$ "gave it its value" would solve the problem of obtaining the source-to-sink shortest path we seek. That is, if we use $P(i, j)$ (where $P$ is mnemonic for "parent") to denote that element $(k, l) \in\{(i-1, j-1),(i-$ $1, j),(i, j-1)\}$ such that the edit path goes from vertex $(k, l)$ to vertex $(i, j)$ in the $(n+1) \times(m+1)$ grid graph that implicitly describes the problem, then all we need to do is store matrix $P$ in split fashion as $P^{\prime}+P^{\prime \prime}$. However, this does not work because it would reveal the edit path to both agents: To get that edit path would require starting at vertex $(n, m)$ and repeatedly following the parent until vertex $(0,0)$ is reached, which appears impossible to do without revealing the path to the agents. To get around this difficulty, we use a different approach that we develop next. 


\subsection{The Backward Version of the Protocol}

The protocol we presented worked by computing (in split form) a matrix $M$ such that $M(i, j)$ contains the length of a shortest path from vertex $(0,0)$ to vertex $(i, j)$ in the grid graph. We call this the forward protocol and henceforth denote the $M$ matrix as $M_{F}$ where the subscript $F$ is a mnemonic for "forward".

One can, in a completely analogous manner, give a protocol that computes for every $(i, j)$ the length of a shortest path from vertex $(i, j)$ to the sink vertex $(n, m)$. We denote the length of such a path as $M_{B}(i, j)$ where the subscript $B$ is a mnemonic for "backward". The edit distance is $M_{B}(0,0)\left(=M_{F}(n, m)\right)$. The protocol for $M_{B}$ is similar to the one for computing $M_{F}$ and is omitted for reason of space limitations (the details will be given in the journal version).

Note that $M_{F}(i, j)+M_{B}(i, j)$ is the length of a shortest source-to-sink path that is constrained to go through vertex $(i, j)$ and hence might not be the shortest possible source-to-sink path. However, if the shortest source-to-sink path goes though vertex $(i, j)$, then $M_{F}(i, j)+M_{B}(i, j)$ is equal to the length of shortest path. We use $M_{C}$ to denote $M_{F}+M_{B}$ (where subscript $C$ is mnemonic for "constrained").

The protocol below finds (in split fashion), for each row $i$ of $M_{C}$, the column $\theta(i)$ of the minimum entry of that row, with ties broken in favor of the rightmost such entry; note that $M_{C}(i, \theta(i))$ is the edit distance $M_{F}(n, m)$. Computing (in split fashion) the $\theta$ function is an implicit description of the edit path:

- If $\theta(i+1)=\theta(i)=j$ then the edit path "leaves" row $i$ through the vertical edge from vertex $(i, j)$ to vertex $(i+1, j)$ (the cost of that edge is, of course, the cost of deleting $\left.\lambda_{i+1}\right)$.

- If $\theta(i+1)=\theta(i)+\delta$ where $\delta>0$ then the client can "fill in" in $O(\delta)$ time the portion of the edit path from vertex $(i, \theta(i))$ to vertex $(i+1, \theta(i)+\delta)$ (because such a "thin" edit distance problem on a $2 \times \delta$ sub-grid is trivially solvable in $O(\delta)$ time). The cumulative cost of all such "thin problem solutions" is $O(m)$ because the sum of all such $\delta$ 's is $\leq m$.

\subsection{Edit Path Protocol}

The steps of the protocol for computing the edit path are:

1. $\mathcal{C}, \mathcal{A}_{1}$, and $\mathcal{A}_{2}$ conduct the edit distance protocol as described in Section 4 to compute $M_{F}$ in split fashion, i.e., $\mathcal{A}_{1}$ gets $M_{F}^{\prime}$ and $\mathcal{A}_{2}$ gets $M_{F}^{\prime \prime}$ such that $M_{F}=$ $M_{F}^{\prime}+M_{F}^{\prime \prime}$.

2. Similarly, $\mathcal{A}_{1}$ and $\mathcal{A}_{2}$ conduct the backward version of the edit distance protocol and compute $M_{B}$ in split fashion. As a result, $\mathcal{A}_{1}$ gets $M_{B}^{\prime}$ and $\mathcal{A}_{2}$ gets $M_{B}^{\prime \prime}$.

3. $\mathcal{A}_{1}$ computes $M_{C}^{\prime}=M_{F}^{\prime}+M_{B}^{\prime}$ and $\mathcal{A}_{2}$ computes $M_{C}^{\prime \prime}=M_{F}^{\prime \prime}+M_{B}^{\prime \prime}$. Note that $M_{C}^{\prime}+M_{C}^{\prime \prime}$ is equal to $M_{C}$.

4. For $i=1, \ldots, n$ in turn, the following steps are repeated:

(a) $\mathcal{A}_{1}$ picks $i$ th row from $M_{C}^{\prime}$, denoted as $\left(v_{0}^{\prime}, \ldots, v_{m}^{\prime}\right)$, and $\mathcal{A}_{2}$ picks $i$ th row from $M_{C}^{\prime \prime}$, denoted as $\left(v_{0}^{\prime \prime}, \ldots, v_{m}^{\prime \prime}\right)$. 
(b) For $0 \leq j \leq m, \mathcal{A}_{1}$ sets $v_{j}^{\prime}=(m+1) * v_{j}^{\prime}$ and $\mathcal{A}_{2}$ sets $v_{j}^{\prime \prime}=(m+1) * v_{j}^{\prime \prime}+$ $(m-j)$; note that $v_{j}^{\prime}+v_{j}^{\prime \prime}=(m+1) * M_{C}(i, j)+(m-j)$. Also observe that, if $M_{C}(i, j)$ is the rightmost minimum entry in row $i$ of $M_{C}$, them $v_{j}^{\prime}+v_{j}^{\prime \prime}$ is now the only minimum entry among all $j \in[0 . . m]$; in effect we have implicitly broken any tie between multiple minima in row $i$ in favor of the rightmost one (which has the highest $j$ and therefore is "favored" by the addition of $m-j$ ). Note, however, that breaking the tie through this addition of $m-j$ without the prior scaling by a factor of $m+1$ would have been erroneous, as it would have destroyed the minima information.

(c) $\mathcal{A}_{1}$ and $\mathcal{A}_{2}$ run the minimum finding protocol for split data (described in [2]) on their respective $\left(v_{0}^{\prime}, \ldots, v_{m}^{\prime}\right)$ and $\left(v_{0}^{\prime \prime}, \ldots, v_{m}^{\prime \prime}\right)$. As a result, $\mathcal{A}_{2}$ gets an $x^{\prime}$ and $\mathcal{A}_{2}$ gets and $x^{\prime \prime}$ whose sum $x^{\prime}+x^{\prime \prime}$ is $\min \left(v_{0}^{\prime}+v_{0}^{\prime \prime}, \ldots, v_{m}^{\prime}+v_{m}^{\prime \prime}\right)$.

(d) $\mathcal{A}_{1}$ and $\mathcal{A}_{2}$ send $x^{\prime}$ and (respectively) $x^{\prime \prime}$ to $\mathcal{C}$. $\mathcal{C}$ computes

$$
\begin{aligned}
p_{i} & =x^{\prime}+x^{\prime \prime} \bmod (m+1) \\
& =\left((m+1) * M_{F}(i, \theta(i))+(m-\theta(i))\right) \bmod (m+1) \\
& =m-\theta(i),
\end{aligned}
$$

therefore obtains $\theta(i)=m-p_{i}$.

5. As mentioned earlier, given $\theta(0), \ldots, \theta(m), \mathcal{C}$ can compute the edit path in $O(m)$ additional time.

Performance Analysis The computation by the client includes initializing the edit distance protocol (step 1) and computing the edit path from the $\theta(i)$ s (step 5). It can be done in $O(m+n)$ time and communication.

The agents run the edit distance protocol twice (steps 1 and 2), and the minimum finding protocol $n$ times (step 4). Each edit distance protocol can be done in $O(\sigma m n)$ time and communication, and each minimum finding protocol needs $O(m)$ time and communication. Therefore, the total computation and communication cost for each agent is $O(\sigma m n)$. The space complexity for each agent is $O(m n)$ as the agents need to store $M_{C}$ in split fashion; in the journal version of this paper, we will include a solution of $O(m+n)$ space complexity for the agents (i.e., same as for the edit distance protocol rather than edit path).

\section{Concluding Remarks}

We gave efficient protocols for a customer to securely outsource sequence comparisons to two remote agents, such that the agents learn nothing about the customer's two private sequences or the result of the comparison. The local computations done by the customer are linear in the size of the sequences, and the computational cost and amount of communication done by the external agents are close to the time complexity of the best known algorithm for solving the problem on a single machine. Such protocols hold the promise of allowing weak computational devices to avail themselves of the computational, storage, and bandwidth resources of powerful remote servers without having to reveal to those servers their private data or the outcome of the computation. 


\section{Acknowledgement}

We would like to thank the anonymous reviewers for their helpful comments.

\section{References}

1. A. V. Aho, D. S. Hirschberg and J. D. Ullman. Bounds on the Complexity of the Longest Common Subsequence Problem. Journal of the ACM 23, 1, pp.1-12 (1976).

2. M. J. Atallah, F. Kerschbaum, and W. Du. Secure and Private Sequence Comparisons. Proceedings of 2nd ACM Workshop on Privacy in Electronic Society (2003).

3. M. J. Atallah, K. N. Pantazopoulos, J. Rice, and E. H. Spafford. Secure Outsourcing of Scientific Computations. Advances in Computers 54, 6, pp.215-272 (2001).

4. P. Beguin and J. J. Quisquater. Fast Server-Aided RSA Signatures Secure Against Active Attacks. CRYPTO'95, pp.57-69 (1995).

5. C. Cachin. Efficient Private Bidding and Auctions with an Oblivious Third Party. Proceedings of the 6th ACM Conference on Computer and Communications Security, pp.120-127 (1999).

6. W. Du and M. J. Atallah. Protocols for Secure Remote Database Access with Approximate Matching. Proceedings of the 1st ACM Workshop on Security and Privacy in E-Commerce (2000).

7. M. Fischlin. A Cost-Effective Pay-Per-Multiplication Comparison Method for Millionaires. RSA Security 2001 Cryptographer's Track, Lecture Notes in Computer Science 2020, pp.457-471 (2001).

8. I. Foster and C. Kesselman, editors. The Grid: Blueprint for a New Computing Infrastructure. Morgan Kaufmann Publishers (1999).

9. O. Goldreich. Secure Multi-party Computation (working draft). Available at http://www.wisdom.weizmann.ac.il/home/oded/public_html/pp.html (2001).

10. S. I. Kawamura and A. Shimbo. Fast Server-Aided Secret Computation Protocols for Modular Exponentiation. IEEE Journal on Selected Areas in Communications, 11(5), pp. 778-784 (1993).

11. G. Landau and U. Vishkin. Introducing Efficient Parallelism into Approximate String Matching and a new Serial Algorithm. Proceedings of the 18-th ACM STOC, pp. 220-230 (1986).

12. C. H. Lim and P. J. Lee. Security and Performance of Server-Aided RSA Computation Protocols. CRYPT0'95, pp. 70-83 (1995).

13. H. M. Martinez (ed.) Mathematical and Computational Problems in the Analysis of Molecular Sequences. Bulletin of Mathematical Biology (Special Issue Honoring M. O. Dayhoff), 46, 4 (1984).

14. W. J. Masek and M. S. Paterson. A Faster Algorithm Computing String Edit Distances. Journal of Computer and System Science 20, pp.18-31 (1980).

15. T. Matsumoto, K. Kato and H. Imai. Speeding Up Secret Computations with Insecure Auxiliary Devices. CRYPTO'88, pp. 497-506 (1988).

16. D. Naccache and J. Stern. A New Cryptosystem based on Higher Residues. Proceedings of the ACM Conference on Computer and Communications Security 5, pp.59-66 (1998).

17. S. B. Needleman and C. D. Wunsch. A General Method Applicable to the Search for Similarities in the Amino-acid Sequence of Two Proteins. Journal of Molecular Biology 48, pp.443-453 (1973).

18. T. Okamoto and S. Uchiyama. A New Public-Key Cryptosystem as Secure as Factoring. EUROCRYPT'98, Lecture Notes in Computer Science 1403, pp.308-318 (1998). 
19. B. Pfitzmann and M. Waidner. Attacks on Protocols for Server-Aided RSA Computations. EUROCRYPT'92, pp. 153-162 (1992).

20. R. L. Rivest, L. Adleman, and M. L. Dertouzos. On Data Banks and Privacy Homomorphisms. In Richard A. DeMillo, editor, Foundations of Secure Computation, Academic Press, pp. 169-177 (1978).

21. D. Sankoff. Matching Sequences Under Deletion-insertion Constraints. Proceedings of the National Academy of Sciences of the U.S.A. 69, pp.4-6 (1972).

22. D. Sankoff and J. B. Kruskal (eds.). Time Warps, String Edits and Macromolecules: The Theory and Practice of Sequence Comparison. Addison-Wesley, Reading, PA (1983).

23. Bruce Schneier. Applied cryptography : protocols, algorithms, and source code in C (Second Edition). John Wiley \& Sons, Inc (1995).

24. P. H. Sellers. An Algorithm for the Distance between two Finite Sequences. Journal of Combinatorial Theory 16, pp.253-258 (1974).

25. P. H. Sellers. The Theory and Computation of Evolutionary Distance: Pattern Recognition. Journal of Algorithms 1, pp.359-373 (1980).

26. E. Ukkonen. Finding Approximate Patterns in Strings. Journal of Algorithms 6, pp.132-137 (1985).

27. R. A. Wagner and M. J. Fischer. The String to String Correction Problem. Journal of the ACM 21,1, pp.168-173 (1974).

28. C. K. Wong and A. K. Chandra. Bounds for the String Editing Problem. Journal of the ACM 23, 1, pp.13-16 (1976).

29. A. Yao. Protocols for Secure Computations. Proceedings of the Annual IEEE Symposium on Foundations of Computer Science 23, pp.160-164 (1982). 